\title{
Point-of-care lung ultrasound in three neonates with COVID-19
}

\section{R. Gregorio-Hernández ${ }^{1}$ (D) - A. B. Escobar-Izquierdo ${ }^{1} \cdot$ J. Cobas-Pazos $^{1} \cdot$ A. Martínez-Gimeno ${ }^{2}$}

Received: 26 April 2020 / Revised: 22 May 2020 / Accepted: 25 May 2020 / Published online: 5 June 2020

(C) Springer-Verlag GmbH Germany, part of Springer Nature 2020

\begin{abstract}
Since March 2020, the world is involved in the COVID-19 pandemic, a disease caused by a novel virus called SARS-CoV-2. Some authors have described the ultrasonographic findings of COVID-19 pneumonia in adults and children, but data on neonates are lacking. Our objective was to describe the ultrasonographic lung pattern on newborns with SARS-CoV-2 infection during the COVID-19 pandemic. Newborns who tested positive for SARS-CoV-2 PCR in respiratory samples and were evaluated with point-of-care lung ultrasound (LU) from March to April 2020 were included. LU was performed bedside by a single investigator at the time of diagnosis and every $48 \mathrm{~h}$ during the first week following diagnosis. Six areas were studied. Three neonates were included. Infants' comorbidities included meconium aspiration syndrome, bronchopulmonary dysplasia, and Hirschsprung's disease. One required mechanical ventilation. No deaths occurred. LU showed B-lines, consolidation, and spared areas. No pneumothorax or pleural effusion was observed

Conclusions: LU could be of value when managing COVID-19 neonates. We describe the findings of lung ultrasound monitoring during the first week following diagnosis in three neonates with SARS-CoV-2 infection.
\end{abstract}

\section{What is known:}

- Lung ultrasound (LU) is a useful tool in COVID-19 management in adults. To date, no report on LU and neonates with SARS-CoV-2 infection has been published.

What is new:

- This study adds evidence about LU findings in neonates with SARS-CoV-2 infection.

Keywords Lung ultrasound $\cdot$ Neonate $\cdot$ COVID-19 $\cdot$ SARS-CoV-2 $\cdot$ Point-of-care ultrasound $\cdot$ Ultrasonography $\cdot$ Coronavirus

$\overline{\text { Communicated by Daniele De Luca }}$

Electronic supplementary material The online version of this article (https://doi.org/10.1007/s00431-020-03706-4) contains supplementary material, which is available to authorized users.

\section{R. Gregorio-Hernández}

rebecagh@gmail.com

A. B. Escobar-Izquierdo anaescobar@movistar.es

J. Cobas-Pazos

mjuliacobas@gmail.com

A. Martínez-Gimeno

amgimeno@sescam.jccm.es

1 Neonatology Area, Pediatrics Department, Virgen de la Salud Hospital, Toledo, Spain

2 Pediatrics Department, Virgen de la Salud Hospital, Toledo, Spain

\section{Abbreviations}

COVID-19 Coronavirus disease 2019

SARS-CoV-2 Severe acute respiratory syndrome coronavirus 2

PCR Polymerase chain reaction

CT scan Computed tomography scan

LU Lung ultrasonography

AAP American Academic of Pediatrics

SENeo Spanish Society of Neonatology

MAS Meconium aspiration syndrome

BPD Bronchopulmonary dysplasia

PMA Postmenstrual age

\section{Introduction}

Since December 2019, a novel virus called SARS-CoV-2 (severe acute respiratory syndrome coronavirus 2 ) has been described as responsible for a life-threatening disease: COVID- 
19 (coronavirus disease 2019) declared by the World Health Organization (WHO) as a pandemic on March 11, 2020 [1].

In the neonatal period, only a few cases have been reported positive, the largest series coming from a report of 33 pregnant woman with SARS-CoV-2 infection and three neonates with a positive polymerase chain reaction (PCR) result in China [2]. These three children had a mild course of the disease except from the one born prematurely. No deaths or vertical transmission have been reported to date.

Lung ultrasonography (LU) has been previously described as a safe tool to study respiratory and cardiac diseases in neonates [3-5]. Some authors have already suggested that LU would be of interest in COVID-19 adult patients at various points of healthcare structure [6-8]. Peng et al. first described the characteristic LU findings in COVID-19 pneumonia in 20 adults, stating that thickening of pleural line, a variety of B-line patterns, and consolidation appeared, together with spared areas. Pleural effusion was uncommon, and A-lines appeared during the recovery phase [9]. These findings were consistent with previous reports on viral pneumonia [10], but, to date, no international consensus on its practical implications has been published. Similar findings have been reported in children [11].

Although some institutions, including the Chinese Neonatal 2019-nCoV Expert Working Group, the American Academic of Pediatrics (AAP), and the Spanish Society of Neonatology (SENeo), had established guidelines to manage this infection in the neonatal population, there is little experience regarding the diagnosis and evolution of the disease in this vulnerable population [12-14]. To our knowledge, there is no previous report about LU findings in newborns developing SARS-CoV-2 infection.

The objective of this study is to describe the ultrasonographic lung pattern in newborns with SARS-CoV-2 infection during the COVID-19 pandemic. We hypothesize that findings in neonates could be similar to those described in adults and children.

\section{Materials and methods}

\section{Study design, setting, and participants}

Consecutive case series including newborns was admitted to a single tertiary neonatal unit with SARS-CoV-2 infection and evaluated by point-of-care LU from March to April 2020. Patients were included if they had a positive result for SARSCoV-2 PCR in a respiratory sample (nasopharyngeal swab or bronchoalveolar aspirate), regardless of their respiratory and clinical situation. Cases were classified following international definitions for fetal, perinatal, and neonatal COVID-19 [15].

Patients were managed according to the SENeo and Spanish Healthcare Ministry guidelines for COVID-19 [13, $16]$ and the unit's protocol. Babies were isolated inside incubators in a separate room with a single nurse in charge, but no negative-pressure room was available. The results of the ultrasounds did not modify the patients' management, as they were performed by a neonatologist not involved in these patients' treatment. Data from the clinical history and informed consent from the parents were obtained.

\section{Lung ultrasound}

Point-of-care lung ultrasound exams were performed by a sole neonatologist experienced in the use of this technique, using a broadband high-frequency linear transducer $(6-11 \mathrm{MHz})$ and the General Electrics LOGIQ 6Q portable ultrasound machine. Depth was adjusted to $4 \mathrm{~cm}$, and focus was set at the pleural line. No harmonics were used. Video images and photographs were taken. The patients were examined inside an incubator, in supine position and at resting state. Swaddling and suckling were offered by the nurse. The ultrasound was repeated every $48 \mathrm{~h}$ during the first week following diagnosis (days $0,+2,+4,+6$ ), maintaining all the safety and cleaning measures established by Spanish Healthcare Ministry guidelines (transducer covered by sterile protection and operator fully equipped with personal protection equipment). Longitudinal and transversal images were obtained. The findings were described qualitatively and semiquantitatively according to previously described Brat's LU score [17]. The LU score ranged from 0 to 18 points (each area with values from 0 to 3 points): 0 points: A-pattern, 1 point: $\geq 3$ B-lines, 2 points: crowded and coalescent B-lines, and 3 points: extended consolidation. Total score ranged from 0 to 18 points. Three areas were studied in each hemithorax:

-Anterior: between medioclavicular and anterior axillary lines.

-Lateral: between anterior and posterior axillary lines.

-Posterior: between paravertebral and posterior axillary lines.

\section{Results}

During the study period, three patients were included. Clinical and epidemiological characteristics of the patients are described in Table 1. Patients 1 and 2 had lung comorbidities: meconium aspiration syndrome (MAS) and bronchopulmonary dysplasia (BPD) respectively. All samples obtained were nasopharyngeal swab except for a bronchoalveolar aspirate in patient 1 (Table 2). This newborn was studied for SARS-CoV-2 infection because of his mother's results (she was tested because of postpartum fever, no respiratory symptoms), and the other two patients were discovered positive during an extended and active investigation including all patients in the unit after the first case (patient 1) was diagnosed, despite their asymptomatic status. The mother of patient 2 was also asymptomatic, despite a positive result. The three patients were classified as "probable postpartum 
Table 1 Clinical and epidemiological characteristics of the patients included

\begin{tabular}{llll}
\hline & Patient 1 & Patient 2 & Patient 3 \\
\hline Birth weight $(\mathrm{g})$ & 3700 & 1135 & 3550 \\
Sex & $\mathrm{M}$ & $\mathrm{M}$ & $\mathrm{M}$ \\
Mode of delivery & Vaginal & Vaginal & Vaginal \\
Previous diagnosis & Severe hypoxic-ischemic encephalopathy, & Prematurity & Hirschsprung's disease \\
& meconium aspiration and multiorganic failure & (28 weeks), BPD & Yes. Parents negative \\
Parents tested & Yes. Mother positive & Yes. Mother positive & 0 \\
Time on MV since birth $(\mathrm{d})$ & 6 & 0 & 0 \\
Time on NIV or nasal cannula since birth $(\mathrm{d})$ & 16 & 70 & Room air \\
Respiratory support at diagnosis & MV & Room air & No \\
Fever & No & No & 3.7 \\
Minimum lymphocyte count $\left(\times 10^{9} / \mathrm{L}\right)$ & 11.4 & 7.9 & $<1$ \\
Maximum CRP $(\mathrm{mg} / \mathrm{L})$ & 57 & - & 0.1 \\
Maximum PCT $(\mathrm{ng} / \mathrm{mL})$ & 0.82 & - & 1000 \\
Maximum ferritin $(\mathrm{ng} / \mathrm{mL})$ & 491 & - & 1891
\end{tabular}

$d$ days, $g$ grams, $M$ male, $B P D$ bronchopulmonary dysplasia, $M V$ mechanical ventilation, $N I V$ non-invasive ventilation, $C R P C$-reactive protein, $m g$ milligrams, $m L$ milliliter, $n g$ nanogram, $P C T$ procalcitonin

acquired neonatal infection" (positive PCR $\geq 48 \mathrm{~h}$ of life in a neonate not tested at birth). No serology study was performed, as it was not mandatory to confirm the diagnosis.

All three had negative PCR result before discharge (obtained 6-8 days after diagnosis) (Table 2). At least three lung ultrasound exams were obtained on each infant during the study period, at 48-h intervals. LU score evolution can be seen in Fig. 1. See electronic supplementary material (ESM) to find some LU videoclips. Findings observed in the sample can be summarized as follows:

\section{Patient 1}

Term newborn requiring mechanical ventilation, nitric oxide, cooling therapy, inotropes, and antiepileptic drugs because of severe hypoxic-ischemic encephalopathy in a MAS context. At the start of the LU follow-up (3 days of life, DOL), despite the MAS diagnosis, no extended consolidation was seen in any areas, but a thin pleural line with conserved lung sliding and no coalescent B-pattern were shown. However, in the evolution, coalescent B-lines and some consolidation appeared in both lateral and posterior areas, diameters ranging from 5 to $24 \mathrm{~mm}$ (Fig. 2, ESM 1). This patient needed mechanical ventilation for the first 6 DOL and afterwards, nasal cannula for 16 more days. Maximum LU score was 10 points at day +4 .

\section{Patient 2}

This was a preterm infant with a postmenstrual age (PMA) of $39+3$ weeks (78 DOL) when SARS-CoV-2 infection was

Table 2 Samples obtained at diagnosis and follow-up of the patients included

\begin{tabular}{llll}
\hline & Patient 1 & Patient 2 & Patient 3 \\
\hline At diagnosis & & & 6 \\
DOL (d) & 2 & $39+3$ & $39+6$ \\
PMA (wk+d) & $38+3$ & Nasopharyngeal swab & Nasopharyngeal swab \\
Sample obtained & Bronchoalveolar aspirate & Positive & Positive \\
PCR result & Positive & & 13 \\
At follow-up & 10 & 84 & $40+6$ \\
DOL (d) & $39+3$ & $40+2$ & 7 \\
PMA (wk + d) & 8 & 6 & Nasopharyngeal swab \\
Days after diagnosis & Nasopharyngeal swab & Nasopharyngeal swab & Negative \\
Sample obtained & Negative & Negative & \\
PCR result & & & \\
\hline
\end{tabular}

$d$ days, $w k$ weeks, $P C R$ polymerase chain reaction 


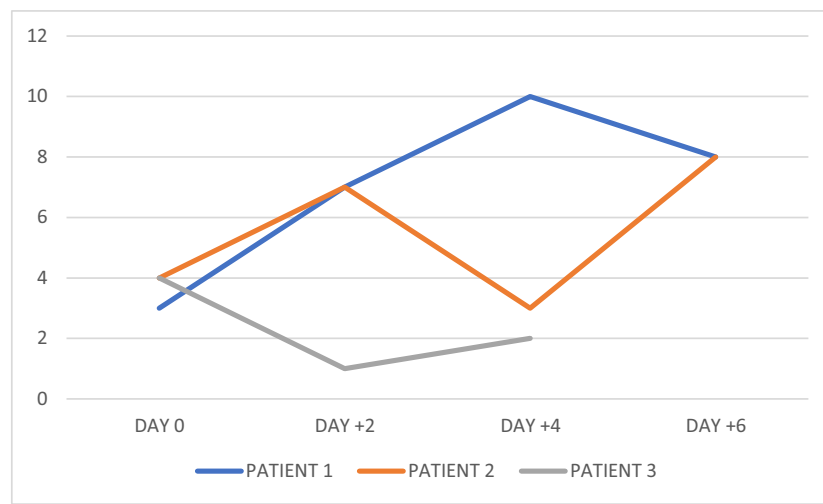

Fig. 1 Lung ultrasound score evolution in the patients included. Each area pointed from 0 to 3 points: 0 points: A-pattern, 1 point: $\geq 3$ Blines, 2 points: crowded and coalescent B-lines, and 3 points: extended consolidations. It was evaluated in 6 areas (anterior, lateral, and posterior). Total punctuation ranged from 0 points to 18 points

confirmed. He was still hospitalized because of oxygen dependence from birth in the context of BPD diagnosis. He had several LU performed before the infection, and, despite showing an improvement from birth, he still presented no coalescent B-pattern with more than three B-lines in all lung areas (Fig. 3). Along the course of the infection, B-pattern turned more crowded, especially in posterior areas, and consolidation appeared in both sides, diameters ranging from 2 to $13 \mathrm{~mm}$ (Fig. 3). The pleural line appeared blurred and thick in almost all areas, but with normal lung sliding. Despite this worsening, he tolerated weaning off respiratory support (nasal cannula) in a few days following the onset of the infection and was discharged home at 85 DOL $(40+3$ PMA $)$ on room air. Maximum LU score was 8 points at day +6 .

\section{Patient 3}

This was a term newborn admitted to the hospital because of Hirschsprung's disease. He did not need any respiratory support during the admission. At diagnosis (6 DOL), LU showed a majority of areas with A-pattern and a thin pleural line with normal lung sliding and isolated B-lines. However, in posterior areas, the pleural line was thick and blurred and B-lines were coalescent in some intercostal spaces. Millimetric "subpleural consolidation" was seen. In the follow-up, these findings rapidly disappeared and the whole lung presented with an A-pattern and a fine pleural line (Fig. 4). Maximum LU score was 4 points at day 0 .

No pleural effusion or pneumothorax was observed in any patient.

\section{Discussion}

We report the lung ultrasonography evolution of three neonates with SARS-CoV-2 infection.

Several reports confirm that it seems to be a mild infection in the majority of cases in children [18-20]. In the neonatal field, evidence is sparse [21]. The SARS-CoV-2 infection can range from asymptomatic infection to severe respiratory distress in neonates and children. Many reports have described CT scan in COVID-19 pneumonia [22]. However, CT scan availability is sparse in several settings, especially in a pandemic context, which implies a lack of resources and requires a rational use of them. There is also a concern regarding ionizing radiation implications in the neonatal population [23].

International guidelines had validated lung ultrasonographic imaging in several neonatal respiratory diseases, as in meconium aspiration syndrome, respiratory distress syndrome, transient tachypnea, etc. [3, 24]. As stated in Chinese and Spanish Societies' recommendations on SARS-CoV-2 infection, $\mathrm{LU}$ is suitable to study even the asymptomatic neonatal patients with a confirmed infection $[12,13]$. However, to date, only a few reports have been published about LU findings in COVID-19, the majority of them being adult case series and clinical recommendations [8]. To our knowledge, there is no report of newborns evaluated with lung ultrasound after testing positive for this new virus. Furthermore, we are not aware of any report addressing the evolution of the ultrasonographic findings in these patients.

Our results show the ultrasonographic lung pattern in three newborns with SARS-CoV-2 infection during this 2019-2020 pandemic. We present three neonates with very different
Fig. 2 Lung ultrasound findings in patient 1. Left, coalescent Bpattern. Right, coalescent Bpattern and consolidation
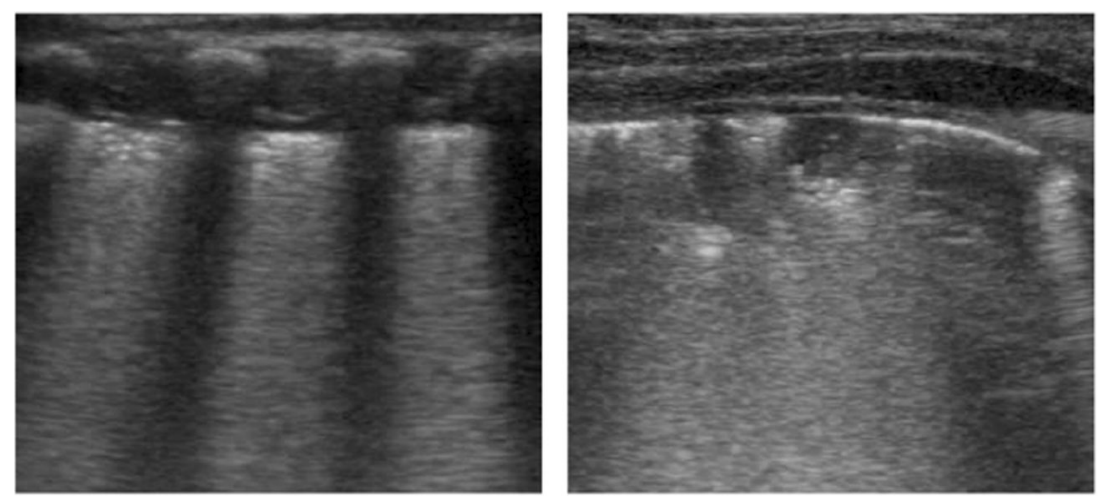
Fig. 3 Lung ultrasound findings in patient 2. Left, irregular pleural line and isolated B-lines. Right, irregular pleural line, coalescent B-lines with spared areas, and a millimetric consolidation
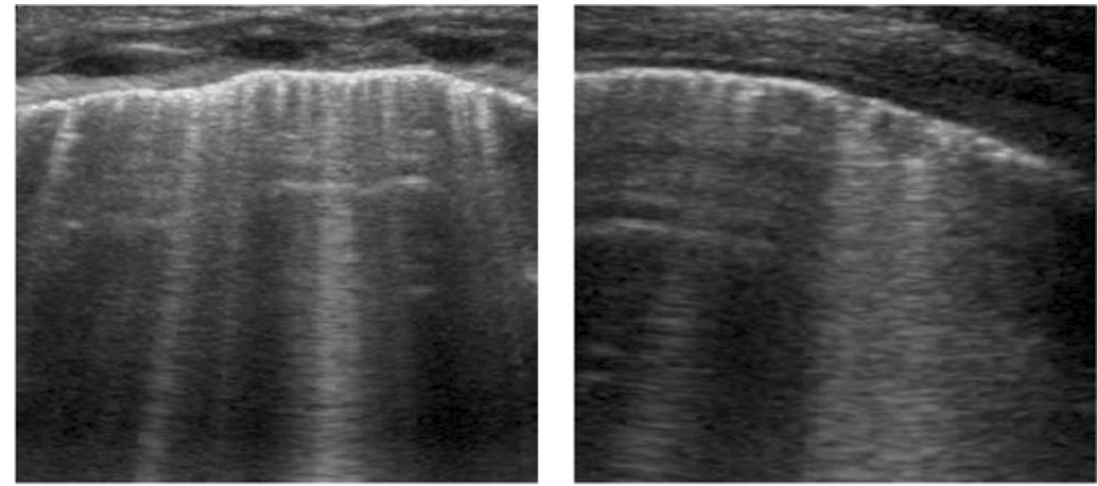

backgrounds, one with an acute lung disease (MAS), another with a chronic lung disease (BPD), and the third patient with no lung disease. This diversity allows for comparison of the evolution of the three. Previous literature has described the ultrasound scan pattern of MAS and BPD [3, 24-26]. We cannot state that the origin of the findings described in our patients is due solely to SARS-CoV-2 infection, but we consider it important to describe the ultrasonographic and clinical course of the infection in these patients, given that newborn with different conditions will suffer from this infection. Surprisingly, the emergence of some consolidation and areas with coalescent B-lines was not accompanied by a respiratory deterioration, in contrast with adult reports [8]. This is important to note, since LU is a clinical tool, always integrated in the clinical context of the patient, and decisions regarding treatment should always keep the clinical condition in mind.

Even the patient without any previous lung disease (patient 3) showed some isolated B-lines. The significance of this finding is worth noting, since it has been reported that even neonates without any respiratory disease can show B-lines up to 19 days after birth [27].

Regarding LU score, all the three patients had an initial score of 3-4 points, proving that some degree of alveolarinterstitial syndrome is very common in newborns. Patients 1 and 2 scores increased to $8-10$ points during the course of the disease process. The patient with the worst respiratory evolution (patient 1) had the highest scores, and patient 3 (no need for respiratory support) had the lowest, although the evolution was different between them (Fig. 1). This score was modified from an index proposed for adult patients. It correlates with extravascular lung water and has been demonstrated useful in predicting mechanical ventilation or surfactant needs in preterm population [28, 29].

Our findings are consistent with those previously described in adults and children: thick pleural line, spared areas, B-lines, consolidation, and no pleural effusion $[11,30]$.

Limitations of our report are that two of the newborns had respiratory diseases in addition to SARS-CoV-2 infection, and this may cause confusion about the significance of the findings. However, we compared the findings with a previous LU in the patient with BPD and also presented a term neonate with no lung disease. The patients described had mild courses of the respiratory disease, seen as consistent with the ultrasonographic appearance. However, it is of interest to study this group of patients due to the possibility of oligosymptomatic infection in infants and neonates [31]. Lung ultrasonography is a feasible complementary test with clear benefits in triage, diagnosis, and management without the risks of patient transfer or ionizing radiation [8]. Further reports are needed to evaluate the usefulness of LU in the study of newborns with COVID-19.

\section{Conclusion}

We show the findings of lung ultrasound monitoring after SARS-CoV-2 infection in the three neonates. The findings are nonspecific, and more evidence is needed to determine the significance of lung ultrasonography in the practical approach to this disease.

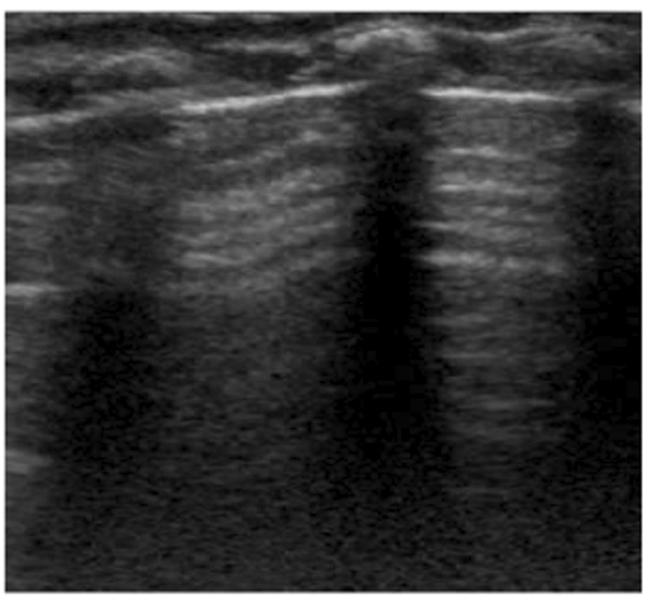

Fig. 4 Lung ultrasound findings in patient 3. Normal pleural line and Alines 
Acknowledgments We would like to thank Almudena AlonsoOjembarrena and Michael P. Smith-O'Brien for their critical review of the text, and all the neonatal nurses for their help, work, and assistance in this project.

Authors' contributions R.G.H. designed the study, performed the acquisition and analysis of the data and drafted the text. AB.E.I., J.C.P. and A.M.G. made contributions to the conception of the work and revised it critically. All the authors revised and approved the final version of the text.

\section{Compliance with ethical standards}

Conflict of interest The authors declare that they have no conflict of interest.

Ethical approval This article does not contain any studies with human participants or animals performed by any of the authors.

Informed consent Informed consent was obtained from the legal guardians of all individual participants included in the study.

\section{References}

1. Cucinotta D, Vanelli M (2020) WHO declares COVID-19 a pandemic. Acta Biomed. 91(1):157-160

2. Zeng L, Xia S, Yuan W et al (2020) Neonatal early-onset infection with SARS-CoV-2 in 33 neonates born to mothers with COVID-19 in Wuhan, China. JAMA Pediatr:e200878. https://doi.org/10.1001/ jamapediatrics.2020.0878

3. Kurepa D, Zaghloul N, Watkins L, Liu J (2018) Neonatal lung ultrasound exam guidelines. J Perinatol. 38(1):11-22

4. Sharma D, Farahbakhsh N (2019) Role of chest ultrasound in neonatal lung disease: a review of current evidences. J Matern Neonatal Med. 32(2):310-316

5. Raimondi F, Yousef N, Migliaro F, Capasso L, De Luca D (2018) Point-of-care lung ultrasound in neonatology: classification into descriptive and functional applications [published online ahead of print, 2018 Jul 20]. Pediatr Res. 20:1-8

6. Soldati G, Smargiassi A, Inchingolo R, Buonsenso D, Perrone T, Briganti DF et al (2020) Is there a role for lung ultrasound during the COVID-19 pandemic? J Ultrasound Med. https://doi.org/10. 1002/jum. 15284

7. Vetrugno L, Bove T, Orso D, Barbariol F, Bassi F, Boero E, Ferrari G, Kong R (2020) Our Italian experience using lung ultrasound for identification, grading and serial follow-up of severity of lung involvement for management of patients with COVID-19. Echocardiography. 37(4):625-627

8. Smith MJ, Hayward SA, Innes SM, Miller A (2020) Point-of-care lung ultrasound in patients with COVID-19-a narrative review. Anaesthesia. https://doi.org/10.1111/anae.15082

9. Peng QY, Wang XT, Zhang LN, Critical C, Ultrasound C, Group S (2020) Findings of lung ultrasonography of novel corona virus pneumonia during the 2019-2020 epidemic. Intensive Care Med. $87: 6-7$

10. Berce V, Tomazin M, Gorenjak M, Berce T, Lovrenčič B (2019) The usefulness of lung ultrasound for the aetiological diagnosis of community-acquired pneumonia in children. Sci Rep. 9(1):17957

11. Musolino AM, Supino MC, Buonsenso D, Ferro V, Valentini P, Magistrelli A et al (2020) Lung ultrasound in children with COVID-19: preliminary findings. Ultrasound Med Biol S03015629(20):30198-30198
12. Wang L, Shi Y, Xiao T, Fu J, Feng X, Mu D et al (2020) Chinese expert consensus on the perinatal and neonatal management for the prevention and control of the 2019 novel coronavirus infection (First edition). Ann Transl Med. 8(3):47-47

13. Recommendations on SARS-CoV-2 infection neonatal management. 6th edition. Spanish Society of Neonatology [Internet]. [cited 2020 Apr 22]. Available from: https://www.seneo.es/images/site/ noticias/home/Recomendaciones_SENeo_SARS-CoV-2Version 50.pdf

14. Newborn care initial guidance. Critical updates on COVID-19. American Academy of Pediatrics [Internet]. [cited 2020 Apr 7]. Available from: https://services.aap.org/en/pages/2019-novelcoronavirus-covid-19-infections/\#ClinicalGuidance

15. Shah PS, Diambomba Y, Acharya G, Morris SK, Bitnun A (2020) Classification system and case definition for SARS-CoV-2 infection in pregnant women, fetuses, and neonates. Acta Obstet Gynecol Scand. 99(5):565-568

16. Recommendations on management of pregnancy and newborn with COVID-19. Spanish Healthcare Ministry [Internet]. [cited 2020 Apr 7]. Available from: https://www.mscbs.gob.es/profesionales/ saludPublica/ccayes/alertasActual/nCov-China/documentos/ Documento_manejo_embarazo_recien_nacido.pdf

17. Brat R, Yousef N, Klifa R, Reynaud S, Shankar Aguilera S, De Luca D (2015) Lung ultrasonography score to evaluate oxygenation and surfactant need in neonates treated with continuous positive airway pressure. JAMA Pediatr. 169(8):e151797

18. Ludvigsson JF (2020) Systematic review of COVID-19 in children shows milder cases and a better prognosis than adults. Acta Paediatr. 109(6):1088-1095

19. Qiu H, Wu J, Hong L, Luo Y, Song Q, Chen D (2020) Clinical and epidemiological features of 36 children with coronavirus disease 2019 (COVID-19) in Zhejiang, China: an observational cohort study. Lancet Infect Dis S1473-3099(20):30198-5

20. Tagarro A, Epalza C, Santos M, Sanz-Santaeufemia FJ, Otheo E, Moraleda $\mathrm{C}$ et al (2020) Screening and severity of coronavirus disease 2019 (COVID-19) in Children in Madrid. Spain. JAMA Pediatr. 8:e201346

21. Lu Q, Shi Y (2020) Coronavirus disease (COVID-19) and neonate: what neonatologist need to know. J Med Virol. https://doi.org/10. $1002 / j m v .25740$

22. Ai T, Yang Z, Hou H, Zhan C, Chen C, Lv W et al (2020) Correlation of Chest CT and RT-PCR testing in coronavirus disease 2019 (COVID-19) in China: a report of 1014 cases. Radiology. 26: 200642

23. Escourrou G, De Luca D (2016) Lung ultrasound decreased radiation exposure in preterm infants in a neonatal intensive care unit. Acta Paediatr. 105(5):e237-e239

24. Liu J, Copetti R, Sorantin E, Lovrenski J, Rodriguez-Fanjul J, Kurepa D et al (2019) Protocol and guidelines for point-of-care lung ultrasound in diagnosing neonatal pulmonary diseases based on international expert consensus. J Vis Exp (145). https://doi.org/ $10.3791 / 58990$

25. Liu J, Chen S-W, Liu F, Wang Y, Kong X-Y, Li Q-P, Huang JJ (2014) BPD, not BPD, or iatrogenic BPD. Medicine (Baltimore). 93(23):e133

26. Piastra M, Yousef N, Brat R, Manzoni P, Mokhtari M, De Luca D (2014) Lung ultrasound findings in meconium aspiration syndrome. Early Hum Dev. 90:S41-S43

27. Chen S-W, Fu W, Liu J, Wang Y (2017) Routine application of lung ultrasonography in the neonatal intensive care unit. Medicine (Baltimore). 96(2):e5826

28. De Martino L, Yousef N, Ben-Ammar R, Raimondi F, ShankarAguilera S, De Luca D (2018) Lung ultrasound score predicts surfactant need in extremely preterm neonates. Pediatrics. 142(3): e20180463 
29. Yousef N, Vigo G, Shankar-Aguilera S, De Luca D (2020) Semiquantitative ultrasound assessment of lung aeration correlates with lung tissue inflammation. Ultrasound Med Biol. 46(5):12581262

30. Pang H, Zhang B, Shi J, Zang J, Qiu L (2019) Diagnostic value of lung ultrasound in evaluating the severity of neonatal respiratory distress syndrome. Eur J Radiol. 116:186-191
31. Canarutto D, Priolo A, Russo G, Pitea M, Vigone MC, Barera G (2020) COVID-19 infection in a paucisymptomatic infant: raising the index of suspicion in epidemic settings. Pediatr Pulmonol. 55(6):E4-E5

Publisher's note Springer Nature remains neutral with regard to jurisdictional claims in published maps and institutional affiliations. 